\title{
Sensing Technologies for Metal Forming
}

\author{
Ming Yang \\ Graduate School of System Design, Tokyo Metropolitan University, \\ 6-6 Asahigaoka, Hino-shi, Tokyo 191-0065, Japan \\ (Received April 10, 2019; accepted June 13, 2019)
}

Keywords: process monitoring, die-embedded sensors, soft sensing, intelligent process visualization

Studies on sensing technologies for metal forming are reviewed in this study. The sensing technologies are divided into four categories: direct and indirect metrologies with commercial sensors, the development of particular sensors or sensing systems, and intelligent metrologies associated with soft sensing. The state-of-the-art sensing technologies in each category are introduced with case studies.

\section{Introduction}

Metal forming is one of the most fundamental processes for manufacturing metallic parts and components with a low cost in industry. With increasing number of requirements for lightweight and high-strength parts, processes for hardly processing materials, such as highstrength steel and titanium and aluminum alloys, are becoming more important. Processes for these materials face various issues, such as reduced formability or higher flow resistance and the need for expert knowledge to design dies and processes. ${ }^{(1)}$ On the other hand, with the spread of the internet and low-cost sensors, initiatives toward realizing an Internet of Things (IoT) society are becoming widespread. A concept called connected industries is attractive for creating effective value-added production systems in manufacturing. ${ }^{(2)}$ The development of processes for hardly processing materials and a reduction in the number of defective products through the use of IoT technology are expected in metal forming industries. However, since dies whose shape corresponds to the product are used in most metal forming processes, the introduction of IoT into metal forming processes faces a major difficulty owing to the complexity of material deformation and the difficulty in visualizing phenomena such as the complicated deformation of materials occurring inside dies and the tribological physics occurring at the interface of the material and dies. An urgent task is to develop sensing technologies to visualize the phenomena in dies as well as process monitoring technologies by fusing various types of sensor information. In this paper, state-of-the-art sensing technologies are reviewed to provide an outlook on metal forming with IoT technology.

*Corresponding author: e-mail: yang@tmu.ac.jp https://doi.org/10.18494/SAM.2019.2399 


\section{Sensing Technologies in Metal Forming}

The sensing technologies used in metal-forming processes can be divided into four categories: direct and indirect metrologies with commercial sensors, the development of particular sensors or sensing systems, such as die-embedded sensors, and intelligent metrologies associated with soft sensing. In this section, some case studies on direct and indirect metrologies with commercial sensors and the development of die-embedded sensors will be introduced. Intelligent metrologies associated with soft sensing will be introduced in the next section.

\subsection{Process sensing by direct metrology}

Typical process monitoring technologies used in metal forming are the measurement of the punch force using a load cell located under the punch and the measurement of the punch stroke using displacement transducers. These are examples of direct measurements by which one can easily obtain information on the deformation resistance of a material and other process states, such as lubrication. A smart die system has been developed for a blanking process by using a compact load cell embedded in a die, which is placed directly under the punch to detect abnormal phenomena, such as blank beating, clogging, and damage to the die, and to provide feedback to the press machine to stop the process on an actual production line. ${ }^{(3)}$ It is also possible to obtain information on the wear of dies from a continuously measured load waveform during processing in the same manner as described above by extracting and comparing features using an AI inference tool. As a result, the life of a die can be predicted from load information obtained by recording the load waveform during punching and comparing it with the initial waveform. The correlation between die wear and the load curve has been obtained from the change in load waveform after tens of thousands of shots by analysis using an AI (deep learning) tool. ${ }^{(4)}$ In another study, the load waveform was used to identify differences in the thickness and material properties of workpieces to accurately predict their springback value in sheet bending. ${ }^{(5)}$ In sheet bending, differences in the thickness and material properties of workpieces and changes in environmental temperature greatly influence the springback characteristic and, as a result, the bending angle varies significantly. At production sites, it is necessary for experts to adjust machines regularly. In Ref. 5, the punch force-stroke curve ( $F-S$ curve) was measured and applied to predict the springback value of the workpiece and control the punch stroke accordingly. Figure 1 shows a schematic configuration of the sensing and control system. The $F-S$ curves for various materials were stored in a database. During the process, the online measured $F-S$ curve was compared with those in the database, the differences in the $F-S$ curves were applied to predict the springback value for process control by using a fuzzy inference engine. Since the $F-S$ curve contains information on the material properties, machine conditions, and ambient temperature, it was used to identify changes in influential factors by comparing the $F-S$ curve measured in-process with those in the database, and then the process was controlled using an $\mathrm{AI}$ inference engine. 


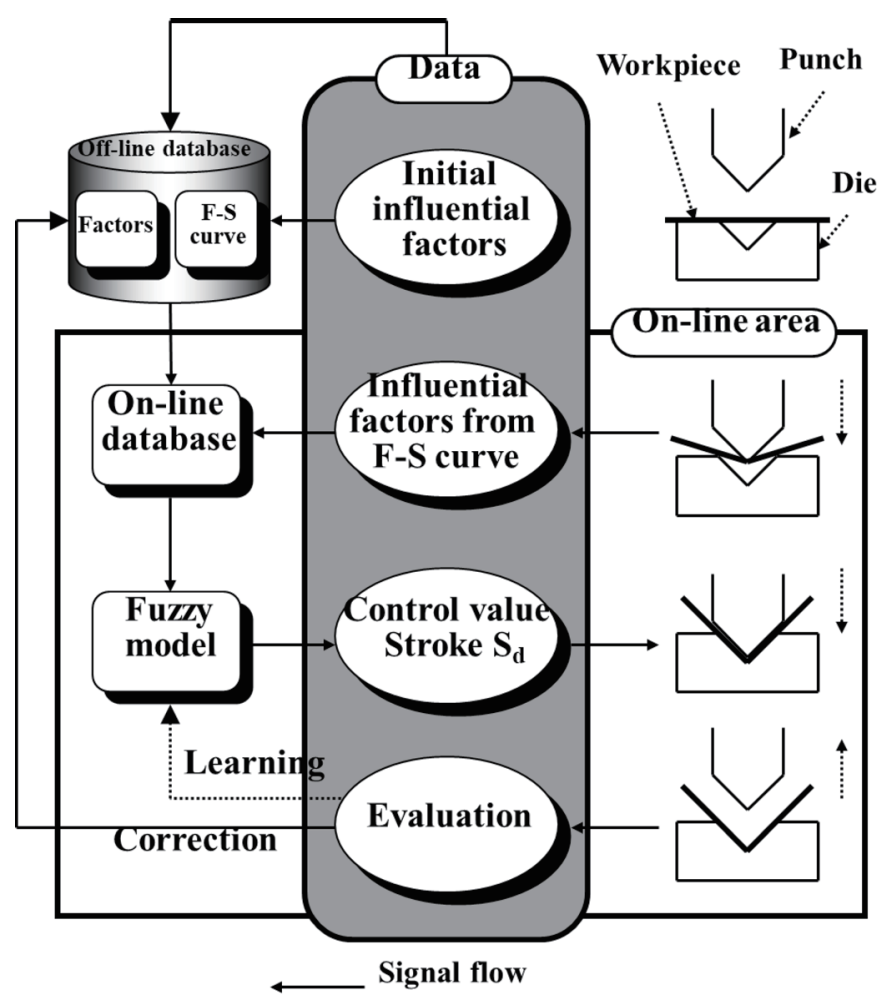

Fig. 1. Configuration of the sensing and control system for the bending process using $F-S$ curves. ${ }^{(5)}$

\subsection{Process sensing by indirect metrology}

Since dies have complicated shapes in most metal forming processes such as stamping and forging, information on the punch force wave is basically average information, which is insensitive to phenomena locally occurring in the dies, such as contact and sliding between the dies and the workpiece. It is necessary to monitor the phenomena occurring during these processes using other sensing technologies, such as die-embedded sensors or indirect metrology.

A bolt-type sensor has been developed by incorporating a sensor into a bolt. A bolt-type sensor can be used by simply fastening a die as an alternative to regular bolts to measure the load variation occurring in dies during processes. Figure 2 shows a cross-sectional view of a bolt-type sensor. ${ }^{(6)}$ In this case, three strain gauges and one temperature gauge are affixed to the bolt, and the axial and vertical loads and bending load can be measured. Moreover, by measuring the temperature at the same time, it is possible to compensate for the load measurement in the case of temperature changes. There is also a type of sensor that incorporates a piezosensor in place of a strain gauge, making it possible to measure the dynamic load characteristics such as vibration at high frequencies. ${ }^{(7)}$ Although it is not easy to separate the various physical phenomena from the sensor information, changes in the information corresponding to abnormal phenomena, as described in the previous section, can be detected sensitively. 


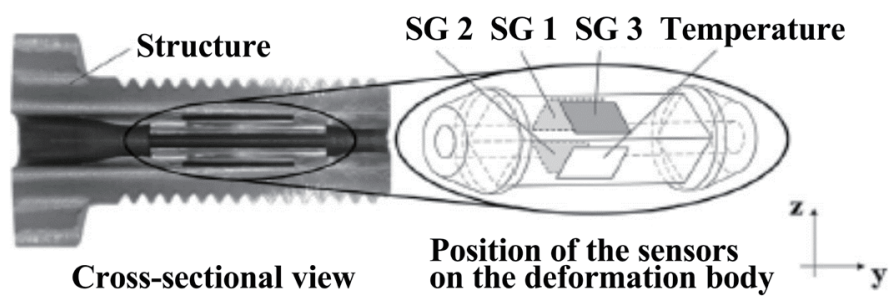

Fig. 2. Bolt-type die-fastening sensor. ${ }^{(6)}$

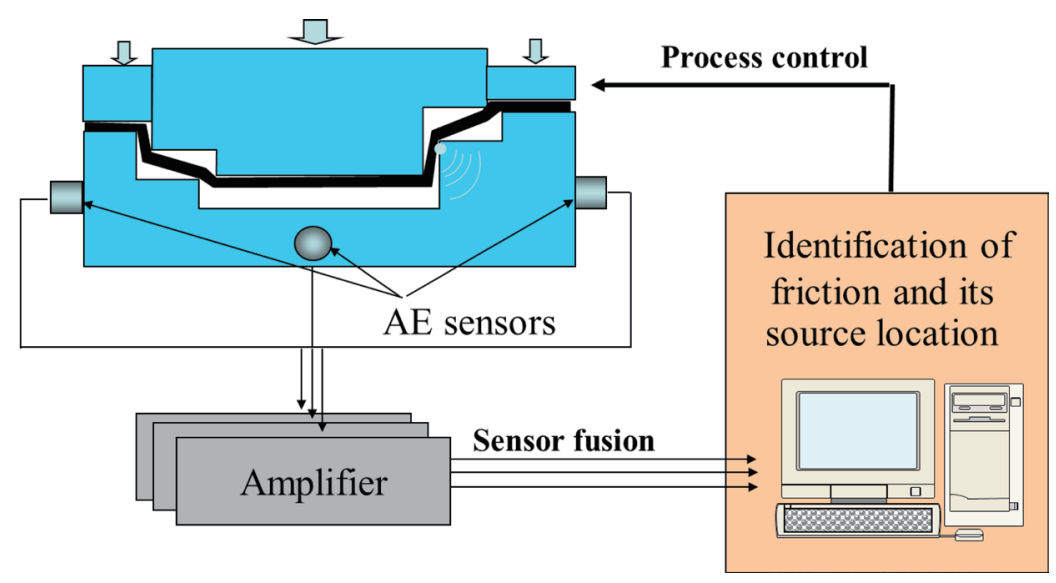

Fig. 3. (Color online) Configuration of AE sensors placed around a die. ${ }^{(8)}$

Another indirect metrology employing acoustic emission (AE) sensors was developed for detecting contact phenomena in dies during stamping. ${ }^{(8)}$ A large acoustic wave is generated in dies when the workpiece comes in contact with the dies or slides between them. Figure 3 shows the configuration of the measurement system. Three AE sensors are placed around a die for deep drawing, and AE sensor signals are monitored during processing and input to a computer. By integrating individual $\mathrm{AE}$ sensor information and analyzing it in an integrated manner, one may detect contact points between the dies and the material as well as the friction state at the interface and the allocation of the friction source. This system can also be applied to detect abnormal phenomena, such as die wearing and breaking, from unexpected AE signals. ${ }^{(9)}$

\subsection{Process sensing using die-embedded metrology}

To obtain information such as the deformation of a workpiece and the friction between the dies and the workpiece in dies with complicated shapes, it is necessary to obtain the relevant information locally by embedding sensors at appropriate locations in a die. A die-embedded sensor system was developed to measure the distribution of the contact force between the workpiece and the dies during stamping or bending. ${ }^{(10)}$ Four high-sensitivity semiconductor 
strain gauges were designed and fabricated on a silicon wafer chip by a semiconductor process, and then a sensor chip was embedded in the forming die under the surface in contact with the workpiece to measure the stress distribution directly during the process. Figure 4 shows the configuration of the sensor and the sensor chip for V-bending. Resistive wires with a length of $0.3 \mathrm{~mm}$ were patterned on a silicon wafer, and the completed sensor chips were cut from the wafer and embedded in the die. Four microgauges were placed closely to each other and connected by a metal wire to construct a Wheatstone bridge. The number of microsensors and their gauge patterns can be appropriately designed according to the measurement target. Figure 5 shows images of a V-bending die embedded with a sensor chip, where the die set on a press-brake machine.

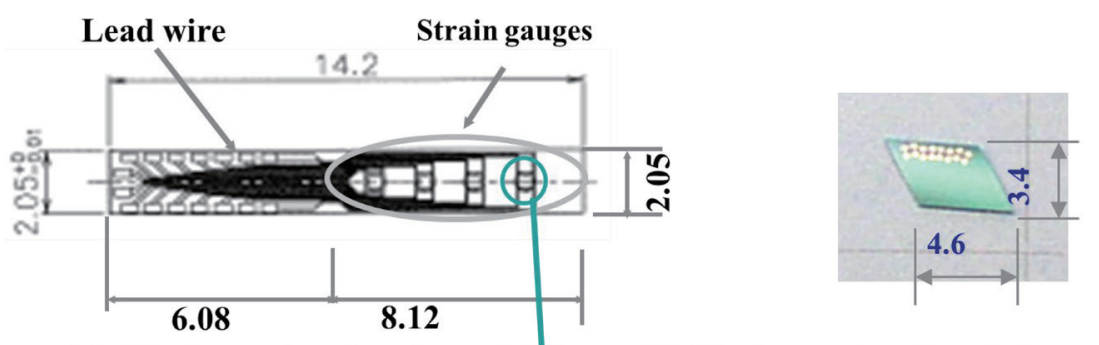

(a) Aligning strain gain units straightly

(b) Aligning strain gain units in are shape

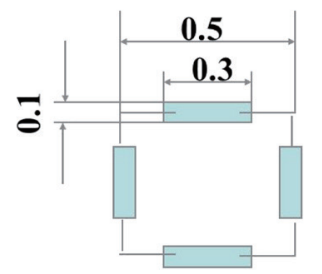

\section{Specifications of strain gauge \\ Dimension : $0.3 \times 0.1 \mathrm{~mm}^{2}$ \\ Resistance : $120 \pm 10 \Omega$ \\ $\mathrm{K}_{\mathrm{G}} \fallingdotseq \mathbf{1 0 0}$}

(c) Strain gauge unit consisting of four strain gains connected by metal wires

Fig. 4. (Color online) Configuration of semiconductor strain gauge sensor chip. ${ }^{(10)}$

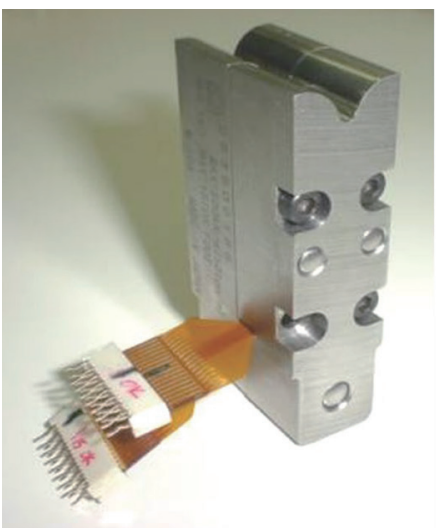

(a)

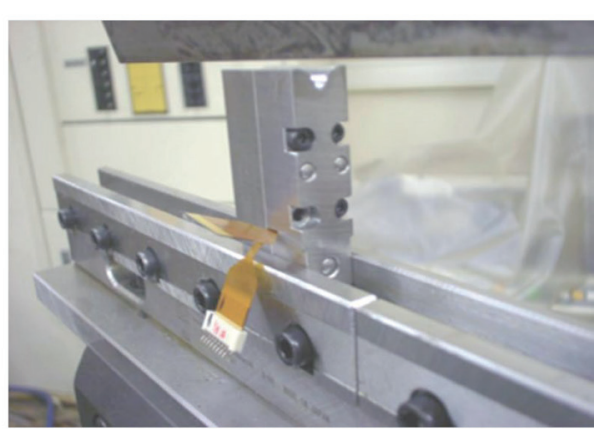

(b)

Fig. 5. (Color online) Images of bending dies with embedded sensing system. (a) Microsensor chip embedded in a bending die. (b) Bending die set on press-brake machine. ${ }^{(10)}$ 


\section{Intelligent Process Sensing and Control by Fusion of Soft Sensing}

It is difficult to realize a complete visualization of the metal forming process using the sensing system described in the previous section since each sensor can only obtain information from where it is located, and it is impossible to sense the material deformation in the whole area or its distribution in dies with complicated shapes only using the sensors. On the other hand, information such as stress/strain and temperature distributions can be determined by computer simulation. Process visualization might be realized by fusing the information obtained by sensing and simulation, which are used to compensate for each other. A conceptual intelligent process with a visualization system that fuses various sensors (hard sensing), process simulation (soft sensing), and databases (knowledge) was proposed for more accurate monitoring metal forming processes by the Japan Society for Technology of Plasticity. ${ }^{(11)}$

In precision forging, finite element (FE) simulation has been applied to evaluate the temperature distribution resulting from the generation of heat due to the plastic deformation of the material during the process. ${ }^{(12)}$ Since the temperature distribution significantly affects the shape accuracy of the product, it is important to control the process to make the temperature as uniform as possible. Figure 6 shows the variation in temperature distribution when different punch speeds are used. The process with only a high punch speed generates a distribution with a high temperature at a portion near the punch corner. However, by reducing the punch speed at the end of the process, the temperature distribution becomes highly uniform. These results imply that a process can be designed to obtain a uniform distribution of the temperature in a deformed workpiece during the process by varying the punch speed in accordance with simulation results. As a result, the accuracy of the product can be greatly improved in comparison with that for the process with a constant punch speed. Since it is easy to program a variable punch speed by using a servo-driven press machine, the optimal design of the process for various punch speeds can improve the formability and accuracy of products especially for hardly processing materials.

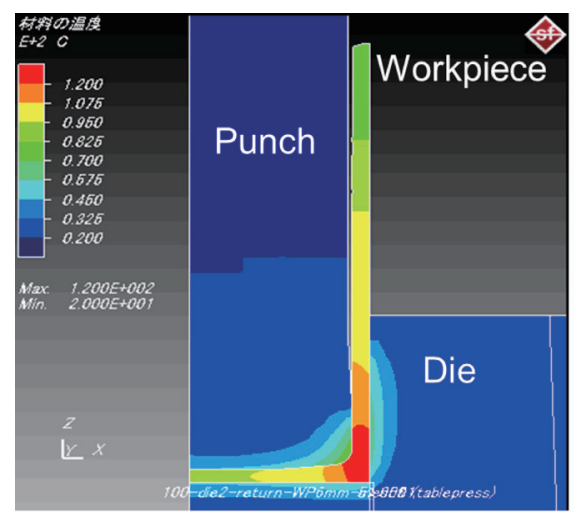

(a)

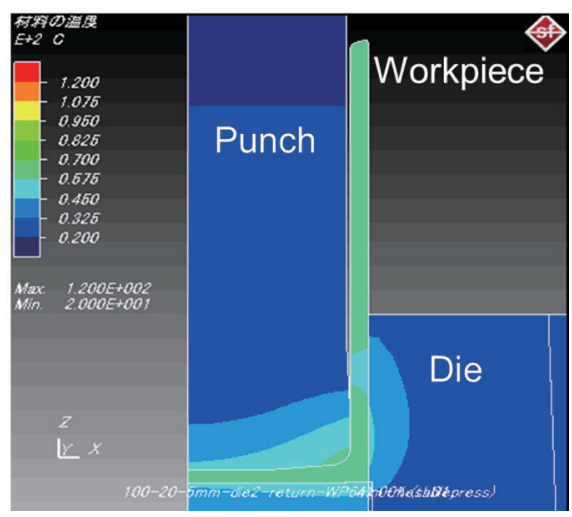

(b)

Fig. 6. (Color online) Temperature distribution with different punch speeds in forging process. ${ }^{(12)}$ (a) Punch speed: $100 \%$. (b) Punch speed: $100 \% \rightarrow 20 \%$. 
Another utilization of computer simulation for soft sensing is the construction of a database for a process control system using results obtained by FE simulation. As described in the last section, a database of monitored $F-S$ curves is useful for process monitoring in metal forming. For a bending process, the database containing more $F-S$ curves for materials with different properties and thicknesses could give more accurate predictions for process control. To meet this requirement, FE simulation can be used to construct databases by varying the parameters using the design of experiment (DOE) method. However, the accuracy and reliability of simulation results may be unsatisfactory since $F-S$ curves are affected by not only the material deformation properties and process conditions but also the deformation of the machine. It is necessary to adjust the simulation results to fit individual machines. A database obtained by the FE analysis of the bending process instead of an experimental database was constructed and a complementary filter was developed for converting the $F-S$ curve to that for an actual bending machine. ${ }^{(13)}$ Figure 7 shows the construction procedure of the database with a complementary filter. A sample sheet material was used for the tensile test and actual bending. The material properties obtained by the tensile test were applied to the FE simulation. The simulated and experimental $F-S$ curves were transformed to a frequency region using a fast Fourier transform (FFT) and the differences among the spectra were used to design the filter. Then, the simulated $F-S$ curves were complemented by the filter and the converted $F-S$ curves to fit the individual press machine. Figure 8 shows the $F-S$ curves before and after conversion. ${ }^{(14)}$ The $F-S$ curve after complementing with the filter matches closely with the experimental curve. It is thus possible to construct the database by FE simulation with a set of material properties designed by the DOE method and a filter designed using the results of a few experiments.

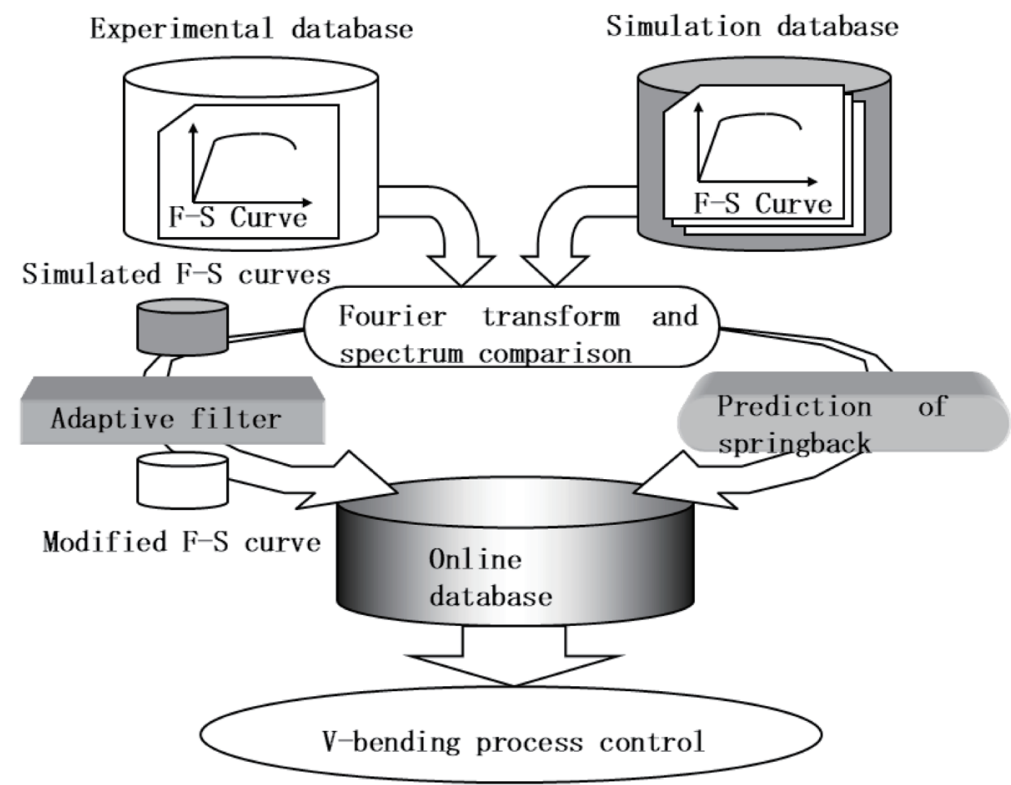

Fig. 7. Construction procedure of database with a complementary filter. ${ }^{(13)}$ 

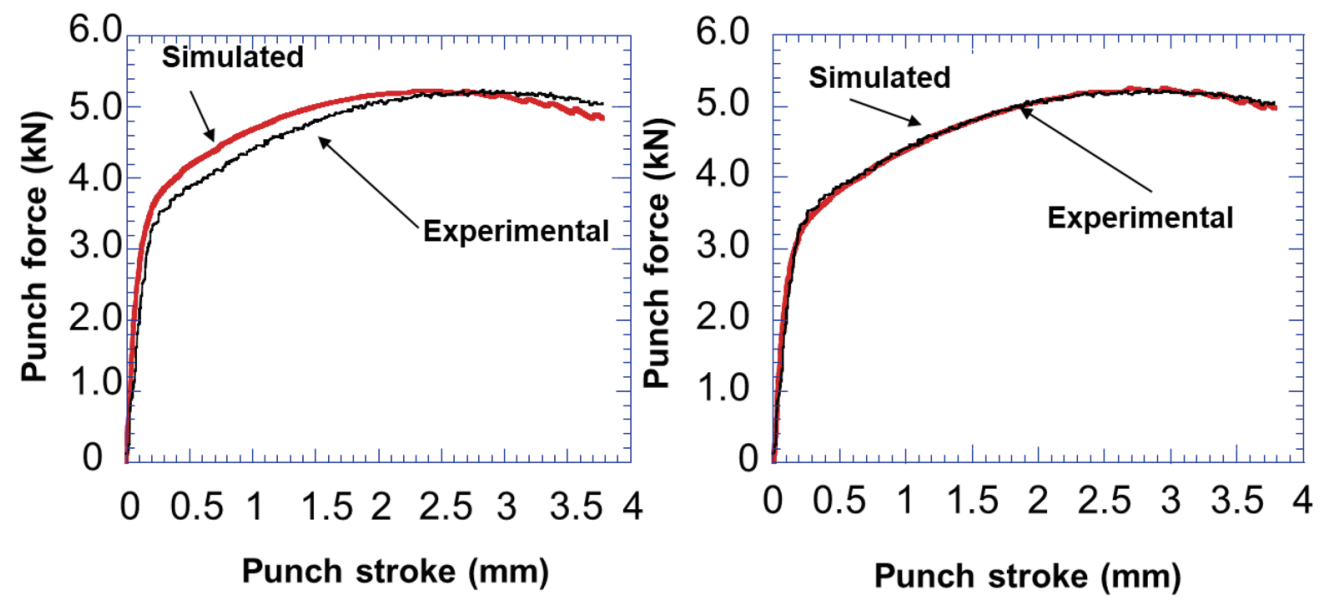

Fig. 8. (Color online) Complemented $F-S$ curves in comparison with experimental curves. ${ }^{(14)}$

\section{Conclusions}

The development of sensing technologies for the visualization of the phenomena in dies and process monitoring has been introduced. The sensing technologies can be divided into four categories according to their metrologies. The sensing technologies can be utilized in actual production for various purposes. They are suitable for production management such as quality control and traceability by integration with network technology. It is becoming easier for factories to realize intelligent press processing and advanced processing on demand to meet needs such as high precision and high efficiency by fusing process visualization technologies.

\section{References}

M. Yang and H. Nishimura: J. Jpn. Soc. Technol. Plast. 55 (2014) 897 (in Japanese).

2 MITI: Connected Industries Tokyo Initiative (2017).

T. Kyuno: SOKEIZAI 59 (2018) 21 (in Japanese).

4 Y. Furuta and H. Aoyama: J. Die Mould Technol. 33 (2018) 92.

5 M. Yang, K. Manabe, and H. Nishimura: J. Mater. Process. Technol. 60 (1996) 249.

6 P. Groche and M. Brenneis: Measurement 53 (2014) 136.

7 S.-Y. Kim, K. Tsuruoka, and T. Yamamoto: Procedia Eng. 81 (2014) 2415.

8 M. Yang, K. Manabe, K. Hayashi, H. Miyazaki, and N. Aikawa: J. Mater. Process. Technol. 139 (2003) 368.

9 K. Saitoh, K. Hayakawa, T. Nakamura, S. Tanaka, and T. Nosue: J. JSTP 47 (2006) 606 (in Japanese).

10 J. Koyama and M. Yang: J. Jpn. Soc. Technol. Plast. 51 (2010) 904 (in Japanese).

11 M. Yang: J. Jpn. Soc. Technol. Plast. 58 (2017) 873 (in Japanese).

12 N. Shinomiya and N. Shirakawa: J. Jpn. Soc. Mech. Eng., Ser. A 79 (2013) 1107.

13 M. Yang, K. Manabe, N. Aikawa, and H. Ogawa: J. Jpn. Soc. Technol. Plast. 39 (1998) 603 (in Japanese).

14 M. Yang, K. Manabe, N. Aikawa, and A. Katayama: J. Jpn. Soc. Technol. Plast. 40 (1999) 996 (in Japanese). 